\title{
TIPO DE PARTO: EXPECTATIVAS DAS MULHERES
}

\author{
Sonia Maria Junqueira Vasconcellos de Oliveira ${ }^{1}$ \\ Maria Luiza Gonzalez Riesco ${ }^{1}$ \\ Claudia Fumiko Rosas Miya ${ }^{2}$ \\ Paula Vidotto ${ }^{2}$
}

Oliveira SMJV, Riesco MLG, Miya CFR, Vidotto P. Tipo de parto: expectativas das mulheres. Rev Latino-am Enfermagem 2002 setembro-outubro; 10(5):667-74.

Estudo descritivo e exploratório, realizado mediante entrevista com 221 puérperas que tiveram parto em maternidades públicas estaduais, localizadas no município de São Paulo. Teve como objetivos: 1. identificar o tipo de parto esperado pelas mulheres, considerando a via vaginal ou cesariana, e sua justificativa; 2. verificar a ocorrência do tipo de parto, segundo as expectativas dessas mulheres; 3. comparar a indicação médica da cesariana com o entendimento das mulheres sobre justificativa dessa intervenção. Os dados revelam que 74,7\% tinham expectativa de que o parto fosse normal, e 25,3\%, de que fosse cesárea. O parto normal, esperado por 165 entrevistadas, ocorreu em 66,1\% dessas mulheres. A justificativa mais citada pelas mulheres para esperar o parto normal foi a recuperação pós-parto mais rápida e, para a cesárea, ter tido cesárea anterior. As justificativas apresentadas por 61 mulheres para a realização da cesariana não era coincidente com a indicação médica em $47,5 \%$ dos casos.

DESCRITORES: parto, cesárea

\section{CHILDBIRTH TYPES: WOMEN'S EXPECTATIONS}

This descriptive and exploratory study was carried out through interviews with 221 puerperal women who gave birth in Sao Paulo State public maternities located within the capital. The purposes of the work were: 1. to identify the type of childbirth women expected to have - whether vaginal births or cesareans - and why; 2. to verify to what extent women's expectations corresponded to the type of birth they had; 3. to compare medical indications for c-sections with women's understanding of justifications they were given for this intervention. Data revealed that $74.7 \%$ of the women expected to have vaginal births and $25.3 \%$ expected to have cesarean sections. Vaginal birth, expected by 165 interviewees, occurred in $66.1 \%$ of these cases. Among women who expected having vaginal births, the most mentioned justification was that recuperation afterwards was faster. Among women who expected cesareans, the most mentioned justification was a previous c-section. The justifications presented by 61 women for having been submitted to c-sections did not coincide with medical indications for this intervention in $47.5 \%$ of the cases.

\section{TIPO DE PARTO: EXPECTATIVAS DE LAS MUJERES}

Estudio descriptivo y exploratorio, realizado mediante entrevista con 221 puerperas que tuvieron el parto en maternidades públicas de en la ciudad de São Paulo. La investigación tuvo como objetivos: 1. identificar el tipo de parto esperado por las mujeres, considerando la vía vaginal o cesárea y su justificación; 2. verificar la ocurrencia del tipo de parto, según las expectativas de esas mujeres; 3. comparar la indicación médica da la cesárea con la comprensión de las mujeres sobre la justificación para esa intervención. Los datos muestran que $74,7 \%$ de las mujeres tenían la expectativa de que el parto fuese normal y 25,3\% que fuese cesárea. El parto normal, esperado por 165 entrevistadas, ocurrió en 66,1\% de estas mujeres. La justificativa más utilizada por las mujeres para esperar el parto normal fue la recuperación postparto más rápida y para la cesárea, haber tenido la cesárea anterior. La justificación presentada por 61 mujeres para la realización de la cesárea no era coincidente con la indicación médica en $47,5 \%$ de los casos.

DESCRIPTORES: parto, cesárea

\footnotetext{
${ }^{1}$ Enfermeira Obstétrica, Professor Doutor, e-mail: soniaju@usp.br; ${ }^{2}$ Enfermeira, Ex-aluna de Graduação em Enfermagem, Ex-bolsista de
} Iniciação Científica da FAPESP. Escola de Enfermagem da Universidade de São Paulo 


\section{INTRODUÇÃO}

O período moderno da obstetrícia inicia-se com a obra de Henrique Deventer, o Novum Lumen Obstetricantibus, publicada em 1701, e com a utilização do fórcipe, por Pedro Chamberlen, em $1677^{(1)}$. Esse período iniciou-se sob a tutela da cirurgia e desenvolveuse sob a óptica que valoriza os aspectos fisiopatológicos da assistência ao parto, em detrimento das dimensões psíquica e cultural que envolvem o nascimento. Como conseqüência, modificou-se o atendimento ao ato de parir, e a gestação e parto, que são fenômenos naturais e fisiológicos, foram transformados em um processo patológico e medicalizado, alterando sua essência original de evento existencial para mãe e filho em acontecimento social.

No Brasil, a prática do parto hospitalar difundiu-se progressivamente, após a segunda guerra mundial, quando novos conhecimentos e habilidades nos campos da cirurgia, anestesia, assepsia, hemoterapia e antibioticoterapia foram sendo incorporados pelos médicos, reduzindo, drasticamente, a morbidade e mortalidade maternas nas intervenções praticadas no parto hospitalar ${ }^{(2)}$. Embora a institucionalização do parto e os avanços tecnológicos tenham proporcionado melhor controle dos riscos materno-fetais, houve incorporação de grande número de intervenções desnecessárias. Ademais, o parto hospitalar afasta a mulher de seu ambiente, colocando-a em local desconhecido e, na maioria das vezes, pouco acolhedor, tornando a experiência do nascimento alienante e desumana ${ }^{(3)}$.

Em 1997, o Grupo de Estudos sobre Nascimento e Parto, do Núcleo de Investigação em Saúde da Mulher e da Criança, do Instituto de Saúde de São Paulo, organizou um workshop com o tema "Humanizando o Nascimento e o Parto". O relatório desse evento apresenta um conceito para humanização do parto, que inclui respeitar a individualidade da parturiente, saber ver e escutar suas necessidades e adequar a assistência segundo a cultura, as crenças e os valores das mulheres. Acrescenta, ainda, que o uso abusivo da tecnologia, com cesarianas desnecessárias, tem contribuído para a desumanização da assistência ao parto. O nascimento por cesariana tornou-se tão comum e disseminado, que a possibilidade de ter um parto normal deixou de ser prática corrente em muitas maternidades, mesmo quando essa é a expectativa da parturiente. Os profissionais nem sempre estão preparados para atender a essa possibilidade, e, ainda que as convicções pessoais da parturiente sejam muito sólidas, o seu desejo nem sempre prevalece ${ }^{(4)}$.

O aumento da freqüência de parto cesárea não apresenta uma associação positiva com o aumento simétrico dos benefícios, para a mãe e recém-nascido. Comparando o risco de morte materna, segundo o tipo de parto, vários estudos nacionais e internacionais revelam maior morbimortalidade materna entre as mulheres submetidas à cesárea, devido a infecções puerperais, acidentes e complicações anestésicas ${ }^{(5)}$.

Estudos norte-americanos apontam aumento, nos índices de cesárea, de 3\%, em 1960, para 23,5\%, em $1990^{(6-7)}$. Dados do Primeiro Simpósio sobre Taxa de Cesárea no Estado de São Paulo, de 1993, apontam freqüência variando de $26,5 \%$ a $76,9 \%$, nas diferentes regiões do Estado. Esses índices elevados e discrepantes impossibilitam uma análise mais rigorosa das reais indicações da cesárea, além de pôr em dúvida o benefício para mãe e filho ${ }^{(2)}$. Dentre as variáveis que influenciam nas altas taxas de cesárea, alegam-se os fatores socioculturais, institucionais e legais, como explicação da preferência pela cesariana, tanto entre as mulheres como entre os médicos ${ }^{(5)}$.

Ainda quanto às causas do elevado número de cesáreas, pode-se mencionar o pouco tempo dedicado pelo médico obstetra para acompanhar o trabalho de parto, em decorrência de atividades concomitantes que desempenha na prática médica, a desinformação da mulher em relação ao parto vaginal, a falta de conhecimento sobre a indicação da cesárea anterior, o despreparo do médico, formado para intervir, e a falta de enfermeiras obstétricas para assistir o parto, a realização da laqueadura tubárea durante a cesariana e questões relacionadas ao pagamento de procedimentos médicos e da analgesia no parto $^{(5,8)}$.

Tendo em vista esse contexto, vários esforços foram tentados para reverter as taxas abusivas de cesariana, se não por completo, pelo menos parcialmente. Algumas medidas foram apresentadas no plano nacional, pelo Ministério da Saúde, como a Portaria $n^{\circ}$ 2.816/98 que instituiu limites máximos de cesárea para o pagamento pelo Sistema Único de Saúde (SUS), inicialmente estabelecidos em até $30 \%$ para o ano de 2000. Para contemplar especificidades locais, o Ministério da Saúde alterou esse critério, com a Portaria $n^{\circ} 466 / 00$, atribuindo às unidades federadas, a definição do limite máximo de cesáreas por instituição. No Brasil, as taxas de cesarianas realizadas no SUS decresceram de $32,5 \%$, no primeiro 
semestre de 1998, para 28\%, em 1999. Matéria do Jornal Folha de São Paulo, publicada em junho de 2000, revela que o percentual de cesarianas realizadas na rede pública, em São Paulo, baixou de 45\% para cerca de 33\%, de 1997 para 1999 , como resultado da Portaria $n^{\circ} 2.816 / 98^{(9)}$.

No modelo intervencionista de assistência obstétrica, a mulher torna-se o objeto da ação, perdendo o controle e a decisão sobre o próprio processo do parto e nascimento ${ }^{(2)}$. A autonomia da mulher no momento do parto está vinculada à sua inclusão na decisão sobre a via de parto; isso ocorre na medida em que ela é informada a respeito das evidências científicas disponíveis para indicação da melhor conduta na situação determinada. Nesse sentido, o diálogo entre o profissional de saúde e parturiente é a base do que se denomina "aliança terapêutica", considerada como uma troca ou negociação para garantir maiores benefícios na assistência, que depende de relações simétricas e não coercitivas entre as partes ${ }^{(10)}$.

A expectativa das gestantes quanto ao tipo de parto está relacionada à maneira como as informações sobre o assunto estão disponibilizadas e acessíveis. Nesse sentido, a orientação durante o pré-natal deve fazer parte da assistência, sendo que um instrumento educativo de alto potencial, conhecido como plano de parto, é ainda pouco desenvolvido em nosso meio. Nesse planejamento, profissionais e usuárias - gestante ou casal - estabelecem vínculos com o serviço de saúde, para determinar onde e por quem o parto será realizado e conhecer as alternativas possíveis na assistência, em situações normais e no caso de surgirem complicações.

Assim, é importante encontrar novas formas que possibilitem à mulher um maior controle sobre o próprio parto, com direito à opção fundamentada na "escolha informada". Isso deve incluir o direito ao suporte emocional, tendo um acompanhante de sua escolha no parto, com quem queira compartilhar essa experiência.

Como enfermeiras, ensinando a assistência e cuidando de parturientes, verificamos que muitas mulheres são submetidas à cesárea, mas desconhecem a indicação de tal procedimento. Além disso, muitas demonstram insatisfação com a falta de oportunidade para expressar suas expectativas e preocupações e tirar suas dúvidas com relação ao parto. Nesse sentido, este estudo se propõe a identificar o tipo de parto esperado pelas mulheres, considerando a via vaginal ou cesariana, e sua justificativa, verificar a ocorrência do tipo de parto, segundo as expectativas dessas mulheres e comparar a indicação médica da cesariana com o entendimento das mulheres sobre justificativa dessa intervenção.

\section{MÉTODO}

Trata-se de um estudo descritivo e exploratório, com abordagem quantitativa. A população foi constituída por uma amostra não-probabilística de puérperas, nos meses de setembro e outubro de 1998, representando, aproximadamente, $3 \%$ das mulheres internadas para parto, em nove hospitais da cidade de São Paulo. Inicialmente, realizou-se levantamento, junto à Secretaria de Estado da Saúde de São Paulo, para identificar as instituições públicas estaduais que tinham leitos obstétricos, no município. Do total de 17 instituições, 11 confirmaram a existência de maternidade, sendo que, em seguida, fezse contato com os hospitais para obter permissão para realizar o estudo. A autorização foi dada por nove instituições, e a coleta de dados teve início após parecer favorável do Comitê de Ética em Pesquisa. Foram incluídas no estudo mulheres que tinham, pelo menos, seis horas de pós-parto, pois, em geral, depois desse período, a puérpera já se encontra em condições de responder às solicitações, sem demonstrar cansaço ${ }^{(3)}$. Após o esclarecimento quanto à finalidade do estudo, foi realizada a entrevista, utilizando-se um formulário. A participação da puérpera ocorreu de forma voluntária, mediante o consentimento livre e esclarecido (Resolução 196/96 do Conselho Nacional de Saúde).

\section{RESULTADOS E DISCUSSÃO}

Participaram 221 mulheres, nas nove instituições que autorizaram a coleta de dados. A idade média das puérperas foi de 25 anos, e o desvio padrão, de 6,05, predominando a faixa etária de 20 a 25 anos (38,5\%). Os dados revelaram baixa escolaridade, com $59,7 \%$ de mulheres com educação fundamental. Quanto à paridade, verificou-se maior freqüência de nulíparas $(41,2 \%)$, seguidas por primíparas (27,6\%), sendo que $15,8 \%$ das mulheres tinham uma cesárea anterior, e 7,2\%, duas ou mais. Do total de puérperas, $96 \%$ receberam assistência pré-natal, 46,2\% freqüentaram entre cinco a sete consultas, e $29,7 \%$ tiveram entre 8 e 13 consultas. Embora a quase totalidade das mulheres tenha realizado o pré-natal, apenas $13,7 \%$ referiram ter recebido informações acerca dos tipos de parto. 
As respostas das mulheres quanto às informações recebidas no pré-natal sobre os tipos de parto sugerem explicações vagas e pouco consistentes, exemplificadas por: "a cesárea é pior que o parto normal"; "não se deve fazer força antes da hora"; "com dores importantes é para procurar o hospital". Pode-se supor que as mulheres, recebendo orientações mais detalhadas, como, por exemplo, as vantagens e desvantagens de cada tipo de parto, poderiam estar instrumentalizadas para discutir e participar, ainda que a relação profissional de saúde-parturiente envolva aspectos complexos, que vão além do poder de argumentação da mulher.

Tabela 1 - Justificativas apresentadas pelas mulheres, quanto ao tipo de parto esperado. São Paulo, 1998

\begin{tabular}{|c|c|c|c|c|c|c|}
\hline \multirow{3}{*}{ (1) Justificativa } & \multicolumn{6}{|c|}{ Parto Esperado } \\
\hline & \multicolumn{2}{|c|}{ Normal } & \multicolumn{2}{|c|}{ Cesárea } & \multicolumn{2}{|c|}{ Total } \\
\hline & $\mathrm{N}$ & $\%$ & $\mathrm{~N}$ & $\%$ & $\mathrm{~N}$ & $\%$ \\
\hline $\begin{array}{l}\text { Recuperação pós- } \\
\text { parto mais rápida }\end{array}$ & 65 & 27,7 & - & - & 65 & 21,8 \\
\hline $\begin{array}{l}\text { Parto mais rápido/ } \\
\text { melhor }\end{array}$ & 50 & 21,3 & 4 & 6,3 & 54 & 18,1 \\
\hline $\begin{array}{l}\text { Experiência do par- } \\
\text { to anterior }\end{array}$ & 43 & 18,3 & 22 & 34,9 & 65 & 21,8 \\
\hline Dói menos & 35 & 14,9 & 7 & 11,2 & 42 & 14,2 \\
\hline $\begin{array}{l}\text { Estava bem na } \\
\text { gravidez }\end{array}$ & 19 & 8,0 & - & - & 19 & 6,4 \\
\hline $\begin{array}{l}\text { Queria que fosse } \\
\text { normal }\end{array}$ & 19 & 8,0 & - & - & 19 & 6,4 \\
\hline É de família & 3 & 1,3 & - & - & 3 & 1,0 \\
\hline É livre de infecção & 1 & 04 & - & - & 1 & 0,3 \\
\hline $\begin{array}{l}\text { Tinha problema na } \\
\text { gravidez }\end{array}$ & - & - & 9 & 14,3 & 9 & 3,0 \\
\hline $\begin{array}{l}\text { Tinha problema com } \\
\text { RN }\end{array}$ & - & - & 8 & 12,7 & 8 & 2,7 \\
\hline $\begin{array}{l}\text { Não tinha pas- } \\
\text { sagem }\end{array}$ & - & - & 4 & 6,3 & 4 & 1,3 \\
\hline $\begin{array}{l}\text { Fazer laqueadura } \\
\text { tubárea }\end{array}$ & - & - & 4 & 6,3 & 4 & 1,3 \\
\hline Pela idade avançada & - & - & 3 & 4,8 & 3 & 1,0 \\
\hline $\begin{array}{l}\text { Medo da morte/de } \\
\text { não ter força }\end{array}$ & - & - & 2 & 3,2 & 2 & 0,7 \\
\hline Total & 235 & 100 & 63 & 100 & 298 & 100 \\
\hline
\end{tabular}

(1) Cada mulher citou uma ou mais justificativas

Os resultados da Tabela 1 evidenciam o predomínio de justificativas com base nas vantagens do parto normal para a mulher, sendo que as mais citadas são ilustradas pelas seguintes falas: "a recuperação é mais rápida", "é mais rápido, melhor" e "porque os outros partos também foram". Merece comentar que algumas mulheres estão bem informadas e têm o conceito de que o parto normal é fisiológico e apresenta-se sem risco para a mãe e bebê, conforme alguns relatos: "porque é menos sofrido, a dor é só na hora e depois passa; a cesárea dói e demora para cicatrizar"; "o parto normal está livre de muita infecção e restabelece mais rápido; a cesárea tem mais risco para a criança e para a mãe". Essas respostas revelam que as mulheres avaliam os riscos e benefícios do parto e estabelecem suas escolhas. $O$ desejo pelo parto normal foi motivado pela expectativa de recuperação rápida e pelo receio de sofrer intercorrências na cesariana que poderiam prejudicar a sua saúde e seu cotidiano no pós-parto. Em outro estudo, justificativas semelhantes a essas, principalmente quanto à "recuperação mais rápida" e "parto normal é mais fácil e rápido", foram encontradas entre primíparas que desejavam o parto normal ${ }^{(11)}$.

Quanto às justificativas para a escolha da cesárea, constam motivos relacionados à mãe e ao bebê, tais como: "o outro parto também foi", "eu tinha problema na gravidez", "cesárea dói menos", "para fazer laqueadura" e "o bebê tinha problema". As justificativas alegadas revelam que o desejo pela cesárea é determinado pelo receio de sofrer dores e pela possibilidade de ter complicações com o recém-nascido e consigo mesma, entre outros fatores. Chama a atenção que as justificativas "parto mais rápido/ melhor", experiência do parto anterior" e "dói menos" são compartilhadas por mulheres que desejavam o parto e por aquelas que desejavam a cesárea. Essa aparente contradição pode ser interpretada a partir da subjetividade e experiência de cada mulher.

Dentre os determinantes socioculturais responsáveis pela preferência das mulheres por cesárea está o medo da dor durante o trabalho de parto e parto. Existe uma crença de que a cesárea eletiva, decidida com antecedência para dia e hora definidos, permitirá à mulher ter um parto sem qualquer dor. Outros fatores que contribuem para o aumento das taxas de cesariana são a cesárea "a pedido da mulher" e para realizar a laqueadura tubária ${ }^{(5)}$.

Dados da autorização de internação hospitalares do SUS de 1995 e 1996 indicam que, cerca de 67\% das cesáreas realizadas teriam a laqueadura como uma indicação camuflada ${ }^{(12)}$. A Organização Mundial da Saúde (OMS) refere que há métodos mais simples e menos arriscados de esterilização tubária, em vez do uso de cesárea para tal fim ${ }^{(13)}$. A lei federal $n^{0} 9.263 / 96$, sobre planejamento familiar, proíbe a ligadura tubária no momento do parto, como forma de cercear essa prática e possibilitar o acesso aos métodos anticoncepcionais no SUS, inclusive a esterilização ${ }^{(14)}$.

Comparando as nulíparas com as mulheres que 
tiveram pelo menos uma cesárea anterior, dentre as primeiras, foi significativamente mais freqüente ter como expectativa o parto normal $(78,4 \%$ e $21,6 \%$, respectivamente; $p<0,001)$. Significativamente $(p<0,001)$, mais mulheres que tiveram experiência com pelo menos um parto normal $(76,4 \%)$ tinham como expectativa ter outro parto normal, comparado com as mulheres que tiveram cesárea anterior $(23,6 \%)$.

Tabela 2 - Tipo de parto esperado pelas mulheres e parto ocorrido. São Paulo, 1998

\begin{tabular}{lcccccc}
\hline & \multicolumn{7}{c}{ Parto Esperado } \\
\hline Parto Ocorrido & $\mathrm{N}$ & $\%$ & $\mathrm{~N}$ & $\%$ & $\mathrm{~N}$ & $\%$ \\
\hline Normal & 109 & 66,0 & 29 & 51,8 & 138 & 62,5 \\
Cesárea & 43 & 26,1 & 24 & 42,8 & 67 & 30,3 \\
Fórcipe & 13 & 7,9 & 3 & 5,4 & 16 & 7,2 \\
\hline Total & 165 & 74,7 & 56 & 25,3 & 221 & 100 \\
\hline
\end{tabular}

Verifica-se, na Tabela 2, que o parto normal foi o que ocorreu com maior freqüência $(62,5 \%)$, seguido pela cesárea $(30,3 \%)$ e pelo fórcipe $(7,2 \%)$. Um dos indicadores que avalia a qualidade do cuidado obstétrico é a taxa de cesariana. No Brasil, a proporção de partos operatórios duplicou entre as décadas de 1970 e 1980, passando de $15 \%$ para $31 \%$; esse aumento está relacionado com a esterilização, pois, em nosso meio, em 1986, 75\% das ligaduras tubárias ocorreram durante a cesárea ${ }^{(15)}$. De acordo com os dados do Ministério da Saúde, em 1996, ocorreram $40,5 \%$ de nascimentos por cesárea no país e $50,3 \%$ no Estado de São Paulo ${ }^{(16)}$. Dados mais atuais mostram que a taxa média de cesárea apresentou queda discreta. Dos três milhões de partos pagos pelos SUS ${ }^{(17)}$, em 1998, o Estado continua apresentado taxa média de cesárea maior $(48,0 \%)$ que a média nacional $(39,0 \%)$. Analisando alguns indicadores de saúde reprodutiva, em países das Américas, no ano de 2000 , constata-se que o Brasil, com taxa de $28 \%$, não é mais o campeão de cesáreas, tendo sido ultrapassado pelo Chile, com 33\% ${ }^{(18)}$.

A taxa de 10 a 15\% de cesárea, definida pela OMS, cobre todas as indicações médicas, sendo que, pelo menos metade das 924.408 cesáreas, equivalentes à taxa de $32,4 \%$ e financiadas pelo Instituto Nacional de Assistência Médica da Previdência Social - INAMPS, em 1994, seriam desnecessárias ${ }^{(19)}$.

Na Tabela 2, nota-se, ainda, que a expectativa para a maioria das mulheres $(74,7 \%)$ era de que o parto fosse normal, atingindo quase o triplo, quando comparado à cesárea (25,3\%). Estudo semelhante, realizado com 71 primíparas na cidade de São José do Rio Preto, resultou que $50,7 \%$ das mulheres tinham expectativa de realizar o parto normal, apesar da "cultura da cesárea" existente naquela cidade, que é conhecida como a campeã desse procedimento no Estado de São Paulo( ${ }^{(13)}$.

Esses dados revelam que não é real a crença de que as mulheres preferem a cesárea ao parto vaginal, pelo menos na população de mulheres estudadas. Os dados mostram que, das 165 mulheres que imaginavam que seu parto seria normal, 109 (66,0\%) tiveram essa expectativa atendida, enquanto que, das $56(25,3 \%)$ que tinham expectativa de cesárea, $29(51,8 \%)$ tiveram parto normal, $24(42,8 \%)$, cesárea, e 3 (5,4\%), fórcipe. No presente estudo, o fórcipe não foi referido como expectativa por nenhuma das mulheres. Em pesquisa realizada em sete hospitais públicos nos Estados de São Paulo e Pernambuco, comparando opiniões das mulheres que tinham, pelo menos, um parto vaginal com aquelas que tinham apenas cesárea, os dados mostraram que foi significativamente mais freqüente considerar como a melhor forma de parto a via vaginal $(90,4 \%$ contra $75,9 \%)$, por aquelas que já tinham tido essa experiência ${ }^{(20)}$, coincidindo com os achados deste estudo.

Tabela 3 - Momento em que foi dada a informação da indicação da cesárea, segundo as puérperas. São Paulo, 1998

\begin{tabular}{lcc}
\hline \multicolumn{1}{r}{ Momento da Informação } & \multicolumn{2}{c}{ Total } \\
& N & $\%$ \\
\hline Antes do parto & 59 & 88,0 \\
Durante o parto & 3 & 4,5 \\
Após o parto & 3 & 4,5 \\
Não recebeu informação & 2 & 3,0 \\
Total & 67 & 100 \\
\hline
\end{tabular}

Observa-se, na Tabela 3, que a maioria das puérperas $(88,0 \%)$ recebeu informação quanto à indicação da cesárea antes da realização do procedimento. Considera-se como não adequado ter recebido informação durante o parto, pois, nesse período, a mulher apresentase em condições desfavoráveis para apreender com clareza tudo o que Ihe é explicado. Conhecer as indicações do parto operatório antecipadamente à sua realização, e não durante ou após o procedimento, permite à mulher expressar seus sentimentos e dúvidas. Mesmo levando em conta que, em algumas situações com indicação de cesárea, existe urgência obstétrica, deixar de informar antecipadamente a mulher que será submetida à cesárea 
é, no mínimo, desconsiderar seu direito como pessoa e violar princípios éticos, refletindo atitude profissional autoritária.

Embora existam evidências favoráveis à presença do acompanhante de escolha da mulher durante o parto, essa recomendação não era adotada nas instituições, campo deste estudo, por ocasião da coleta dos dados. Ter alguém a seu lado, quando uma cesariana ou outra intervenção é necessária, pode ajudar a parturiente na interlocução com a equipe. Em caso de a mulher estar inconsciente por alguma complicação, o familiar ou acompanhante deve ser informado a respeito da conduta a ser seguida.

Estudo sobre a comunicação entre a parturiente e a equipe obstétrica constatou que a falta de informações adequadas e necessárias durante a assistência prestada ou depois dela, pelos profissionais, foi percebida pela mulher como descaso, desamparo e desrespeito, provocando sentimentos de indignação ${ }^{(21)}$.

Na relação profissional de saúde-parturiente acabase repetindo o modelo de relação de subordinação do profissional à instituição de saúde, que é de dominação, pressão e achatamento. Como resultado desse conflito, surgem o autoritarismo e a prepotência, restando à mulher a submissão ou deixar de ser atendida ${ }^{(22)}$. Associado a isso, é comum a equipe de saúde utilizar termos técnicos com linguagem incompreensível para a parturiente.

Tabela 4 - Indicação médica para realizar a cesárea e concordância com justificativa materna. São Paulo, 1998

\begin{tabular}{|c|c|c|c|c|c|c|}
\hline \multirow{3}{*}{ Indicação e Justificativa } & \multirow[t]{2}{*}{ Médica $^{(1)}$} & \multicolumn{5}{|c|}{ Materna $^{(2)}$} \\
\hline & & \multicolumn{2}{|r|}{ Concorda } & \multicolumn{3}{|c|}{ Não Concorda } \\
\hline & $\mathrm{N}$ & $\%$ & $\mathrm{~N}$ & $\%$ & $\mathrm{~N}$ & $\%$ \\
\hline Cesárea anterior & 19 & 24,3 & 5 & 14,7 & - & - \\
\hline Sofrimento fetal & 17 & 21,7 & 7 & 20,6 & 2 & 6,9 \\
\hline Apresentação pélvica & 10 & 12,8 & 8 & 23,6 & 2 & 6,9 \\
\hline Distocia funcional & 8 & 10,3 & 2 & 5,9 & 5 & 17,2 \\
\hline Desproporção cefalopélvica & 7 & 9,0 & 4 & 11,8 & 11 & 38,0 \\
\hline Hipertensão arterial & 5 & 6,4 & 5 & 14,7 & 2 & 6,9 \\
\hline Pós-datismo & 4 & 5,1 & 1 & 2,9 & 1 & 3,4 \\
\hline Macrossomia fetal & 2 & 2,6 & - & - & - & - \\
\hline Descolamento prematuro placenta & 2 & 2,6 & - & - & - & - \\
\hline Laqueadura tubária & - & - & - & - & 2 & 6,9 \\
\hline Oligoâmnio & 1 & 1,3 & 1 & 2,9 & - & - \\
\hline Prolapso de membro & 1 & 1,3 & 1 & 2,9 & - & - \\
\hline${ }^{(3)}$ Outras & 2 & 2,6 & - & - & 4 & 13,8 \\
\hline Total & 78 & 100 & 34 & 100 & 29 & 100 \\
\hline
\end{tabular}

(1) Cada prontuário registrava uma ou mais indicação; 2 prontuários sem registro

(2) Cada puérpera referiu uma ou mais justificativa múltiplas; 6 puérperas não

souberam justificar

(3) Indicação médica: gemelaridade e rotura uterina; justificativa materna: "problemas de bexiga", bebê prematuro, bebê atravessado e bebê mal posicionado
Constata-se, na Tabela 4, que as indicações médicas mais freqüentes para realizar a cesárea foram a cesárea anterior, seguida por sofrimento fetal e apresentação pélvica. Analisando o tipo de parto anterior, verificou-se que 16 mulheres tinham duas ou mais cesáreas. A indicação da cesárea por cesárea anterior é questão polêmica entre os pesquisadores, que, juntamente com o sofrimento fetal e a apresentação pélvica, tem contribuído para o aumento da taxa de cesariana $^{(23)}$.

Os resultados apontam que 34 justificativas para a cesariana, dadas pelas puérperas, estavam de acordo com a indicação encontrada no prontuário médico. Dentre as justificativas maternas concordantes mais citadas pelas puérperas, estavam "bebê estava sentado" (8 puérperas) e "bebê fez cocô" (7 puérperas). As justificativas maternas que apresentaram maior freqüência de discordância foram "sem passagem" (11 puérperas) e "sem dilatação" (5 puérperas).

Merece comentar que duas mulheres referiram, como justificativas da cesárea, a laqueadura tubária. Entretanto, quando analisados seus prontuários, observouse que a indicação anotada pelo obstetra era macrossomia fetal e cesárea iterativa. Chama a atenção que, embora seis mulheres tenham referido desconhecer a indicação da cesárea, somente duas disseram que não receberam essa informação, conforme apresentado na Tabela 3.

Pesquisa realizada em Glasgow, Escócia, com 100 puérperas, mostrou que, três meses após a cesárea, $16 \%$ das mulheres tinham idéia apenas parcial da indicação da cirurgia, e $20 \%$ desconheciam a indicação. A justificativa para esse fato pode ser o momento inadequado em que a explicação foi fornecida à mulher, dificultando o seu entendimento, ou que as informações não eram compreensíveis ${ }^{(24)}$. Convém mencionar que ignorar a indicação de cesárea anterior pode dificultar a condução para um parto normal posterior. Resta saber, ainda, até que ponto a informação referente à indicação da cesárea é valorizada pela equipe de saúde.

No estudo mencionado anteriormente, entre mulheres do interior paulista, a autora considera que inúmeras primíparas foram persuadidas a optar pela cesárea nas consultas de pré-natal, recebendo informações equivocadas sobre os motivos para a realização da cesariana. 
Destaca-se que a legislação que dispõe sobre os direitos dos usuários dos serviços e das ações de saúde no Estado de São Paulo, garante o direito de receber informações claras, objetivas e compreensíveis sobre diagnósticos realizados e riscos, benefícios e inconvenientes das medidas terapêuticas propostas, dentre outros, além do consentimento ou recusa dessa terapêutica, após esclarecimentos adequados ${ }^{(25)}$.

Finalmente, embora a falta de concordância entre a indicação médica da cesárea e o conhecimento da mulher sobre essa indicação possa ter diversas interpretações, reiteramos a possibilidade de existir "mais de uma versão" para justificar a realização de uma intervenção cirúrgica. Os dados desta pesquisa apontam que, lamentavelmente, 14 mulheres foram submetidas a cesárea por terem cesárea prévia, e 10, devido a sofrimento fetal, dentre outras situações, sem terem-se apropriado da justificativa médica para essa intervenção.

\section{CONCLUSÃO}

Os dados permitem concluir que o parto normal era o tipo de parto esperado pela maioria das mulheres $(74,7 \%)$, sendo que essa expectativa diminui dentre aquelas que tinham cesárea prévia. A principal justificativa para preferir a via vaginal para o parto foi a recuperação mais rápida. O parto normal ocorreu em $66,0 \%$ das mulheres que tinham essa preferência, e a cesárea, em 42,8\% que esperavam esse tipo de parto. Além disso, constatou-se que a justificativa dada por 61 mulheres para a realização da cesariana foi discordante da indicação médica em $47,5 \%$ dos casos.

\section{REFERÊNCIAS BIBLIOGRÁFICAS}

1. Delascio D, Guariento A. Obstetrícia normal Briquet. $3^{a}$ ed. São Paulo (SP): Sarvier; 1981.

2. Tanaka ACA. Maternidade: dilema entre nascimento e morte. São Paulo (SP): Hucitec; 1995.

3. Junqueira SM. Sentimentos, percepções e necessidades da parturiente na sala de parto. São Paulo(SP): Escola de Enfermagem da USP; 1987.

4. Rattner D, Hotimsk SN, Venâncio SI, Miranda MM, organizadoras. Workshop humanizando o nascimento e parto. São Paulo (SP): Instituto de Saúde; 1997.

5. Faúndes A, Cecatti JG. A operação cesárea no Brasil. Incidência, tendências, causas, conseqüências e propostas de ação. Cad Saúde Pública 1991 abril/junho; 7(2):150-73.

6. Davis LG, Riedmann GL, Sapiro M, Minogue JP, Kazer RR. Cesarean section rates in low-risk private patients managed by certified nurse-midwives and obstetricians. J Nurse Midwifery 1994 March/April; 39(2):91-7.

\section{CONSIDERAÇÕES FINAIS}

A crescente hospitalização do parto foi estimulada com a argumentação da segurança e melhores recursos tecnológicos existentes no hospital. Atualmente, várias iniciativas têm sido propostas, tentando melhorar a qualidade da assistência ao parto, com um modelo menos intervencionista e assistência mais humanizada. Os caminhos apontados têm sido a criação de centros de parto normal e o resgate da participação da obstetriz e enfermeira obstétrica no processo do parto e nascimento.

Conforme ressalta o Ministério da Saúde, no tipo de parto estão implicados necessidades, riscos e benefícios, complicações e repercussões futuras, sendo importante a informação e formação de opinião entre as mulheres, para que elas possam reivindicar aquilo que é melhor para a sua saúde e a de seus filhos, pois, embora profissionais e mulheres façam a opção antecipada do tipo de parto, esse fato não pode ser visto como uma simples questão de preferência ${ }^{(18)}$.

Vale mencionar que a coleta de dados em prontuários e entrevistas, sem a observação direta da assistência prestada durante o parto, é uma limitação do presente estudo, pois não existe a possibilidade de confrontar as diferentes informações, permitindo uma interpretação que incorpore as várias dimensões envolvidas no contexto.

\section{AGRADECIMENTO}

À Fundação de Amparo à Pesquisa do Estado de São Paulo - FAPESP, pelo apoio financeiro.

7. Sakala C. Midwifery care and out-of-hospital birth sittings: how do they reduce unnecessary cesarean section births? Soc Sci Med 1993 November; 37(10): 1233-50.

8. Zugaib M. A via de parto. [Editorial]. Rev Ginecol Obstet 1990 abril/junho; 1(2):1.

9. Silva $\mathrm{E}$. Cai número de cesarianas na rede pública. Folha de São Paulo 2000 junho 17: Caderno Cotidiano; C5.

10. Diniz CSG. Entre a técnica e os direitos humanos: possibilidades e limites da humanização da assistência ao parto. [tese]. São Paulo (SP): Faculdade de Medicina/USP; 2001.

11. Tavares BB. Expectativa de primíparas de São José do Rio Preto quanto ao tipo de parto e o conhecimento da indicação da cesárea. [dissertação]. São Paulo (SP): Escola de Enfermagem/USP; 2000.

12. Venancio SI. Parto normal "a pedido". [Editorial] Notas sobre Nascimento e Parto 1998; 3(5).

13. World Health Organization. Appropiate technology for birth. Lancet 1985; 2(8452):436-7. 
14. Sorrentino SR. A assistência às mulheres no SUS do Estado de São Paulo. Anais do $1^{\circ}$ Seminário Estadual sobre o Ensino de Enfermagem para a Assistência ao Nascimento e Parto; 1998 outubro 15-16; Ribeirão Preto; São Paulo; 1998. p. 53-9.

15. Berquó E. Brasil, um caso exemplar: anticoncepção e parto cirúrgico à espera de uma ação exemplar. In: Ávila MBM, Berquó E. Direitos reprodutivos: uma questão de cidadania. Brasília: Centro Feminista de Estudos e Assessoria; 1994. p.27-49.

16. DATASUS. Nascidos vivos - Brasil: 1996. [online] 1996. [citado em 8 jan 1999]. Disponível em URL: http:// www.datasus.gov.br/cgi/sinasc/nvmap.htm

17. DATASUS. Nascidos vivos - Brasil: 1998. [online] 1998. [citado em 23 fev 2002]. Disponível em URL: http:// www, datasus.gov.br/cgi/sinasc/nvmap.htm

18. Ministério da Saúde (BR). Secretaria de Políticas de Saúde. Área Técnica de Saúde da Mulher. Parto, aborto e puerpério: assistência humanizada à mulher. Brasília (DF); 2001. p. 32-7.

19. Volochko A. As mulheres exigem cesárea... Anais do $1^{\circ}$ Seminário sobre Nascimento e Parto no Estado de São Paulo; 1996 12-14 julho; São Paulo; 1996.

20. Osis MJD, Pádua KS, Duarte GA, Souza TR, Faúndes A. The opinion of brazilian women regarding vaginal labor and cesarean section. Int J Gynecol Obstet 2001; 75:S59-S66.

21. Caron OAF. Parturiente e equipe obstétrica: a difícil arte da comunicação. [dissertação]. São Paulo (SP): Escola de Enfermagem/USP; 1998.

22. Maldonado MTP, Canella P. A relação médico-cliente em ginecologia e obstétrica. $2^{\text {a }}$ ed. São Paulo (SP): Roca; 1988. 23. Marques CP, Arakaki L, Belfort P. Operação cesariana - a propósito de suas indicações e índices. Femina 1989 abril; 17(4):290-302.

24. Hillan EM. Issues in the delivery of midwifery care. J Adv Nurs 1992 March; 17(3):274-8.

25. Lei $n^{\circ} 10.241$, de 17 de março de 1999. Dispões sobre os direitos dos usuários dos serviços e das ações de saúde no Estado e dá outras providências. Diário Oficial do Estado de São Paulo, São Paulo, 18 mar. 1999. Seção 1, p.1. 